\title{
Isomorfismo tra piani di traslazione di Marshall Hall.
}

\author{
Memoria di Gianfranco Panelia (a Parma)
}

Sunto. - Si studia la suddivisione in classi di equivalenza, rispetto alla relazione di isomor. fismo geometrico, dell'insieme dei quasicorpi di $M$. Hall il nucleo dei quali non sia ismorfo a un suo sottocampo proprio. Si determina, inoltre, il gruppo delle collineazioni spettante ai piani di traslazione che rappresentano quelle ciassi.

Nel presente lavoro determino una condizione caratteristica per l'isomorfismo tra piani di traslazione sopra quasicorpi di MARSHaLL HALL ([2] (1), Appendice II) e il gruppo degli automorfismi spettante a tali piani, nella ipotesi che il campo $F$ che genera il quasicorpo di HaLL abbia almeno quattro elementi, non sia isomorfo a un suo sottocampo proprio e, quindi, risulti necessariamente perfetto. Proseguo, così, uno studio iniziato in una precedente nota $[5]\left({ }^{2}\right)$ : in essa ho gia stabilito che due piani di traslazione sopra due quasicorpi di M. Hall sono sempre isomorfi se $i$ due quasicorpi che li definiscono sono costruiti a partire da un campo isomorfo a un campo di Galois di caratteristica diversa da due o isomorfo al campo reale.

Il lavoro si suddivide in tre parti.

Nella prima di esse (nn. 1 e 2) mi occupo di piani di traslazione relativi a congruenze di sottospazi $(n-1)$-dimensionali dello $S_{2 n-1}$ lineare sopra un corpo $K\left({ }^{3}\right)$ : stabilisco, cosi, due lemmi i quali possono avere interesse, indi. pendentemente dall' uso che di essi si fa nel seguito, per affrontare lo studio della relazione di isomorfismo tra piani di traslazione più generali di quelli da me trattati.

Successivamente, nella seconda parte (nn. 3 e 4), specializzo il corpo $K$ in un campo $F$, che sia diverso dal campo di GaLoIs di ordine due e tale che esista un polinomio di secondo grado $x^{2}-b x-c$ con coefficienti in $F$ o irriducibile su $F$, e considero la congruenza di rette dello $S_{3}$ lineare sopra il campo $F$ atta a definire un piano di traslazione $S(F, b, c)$ relativo al quasicorpo di M. HALL $J(F, b, c)$; faccio vedere, tra l'altro, che a tale congruenza rimane spontaneamente associata una congruenza lineare di rette $\bar{H}(F, b, c)\left({ }^{4}\right)$,

(1) I numeri tra [ ] rinviano alla biblingrafia in fine. Per la definizione di quasicorpo di M. HaLL si può anche consultare il n. 3 di questo lavoro.

(2) Una dimostrazione della proposizione del n. 1 di tale nota è gia data in [7] (pp. 96-97).

(3) Per constatare che a un piano di traslazione, che sia di dimensione finita sopra il suo nuoleo, si può associare una congruenza di spazi $(n-1)-$ dimensionali dello $S_{2 n-1}$ lineare sopra u'n corpo isomorfo al nucleo del piano di traslazione, si confronti [1].

(4) Che risulta ellitica nel caso che il polinomio $x^{2}-b x-c$ sia separabile su $F$. 
la quale individua un piano di traslazione isomorfo al piano lineare affine sopra il campo estensione algebrica di $F$ mediante il polinomio $x^{2}-b x-c$.

Ciò premesso, al n. 5, dimostro che se il campo $F$ ha almeno quattro elementi, se non è isomorfo a un suo sottocampo proprio, e se, quindi, $\grave{e}$ necessariamente perfetto, condizione necessaria e sufficiente perchè $i$ piani $S(F, b, c)$ e $S\left(F, b^{\prime} ; c^{\prime}\right)$ siano isomorfi è che le congruenze lineari ellittiche di rette $\bar{H}(F, b, c)$ e $\bar{H}\left(F, b,{ }^{\prime} c^{\prime}\right)$ ad essi associate risultino proiettivamente equivalenti $\left({ }^{5}\right)$.

Infine, al n. 6, immaginando lo $S_{3}$ come spazio improprio dello $S_{4}$ lineare affine sopra il campo $F$, provo che se il campo $F$ ha almeno quattro elementi, se non è isomorfo a un suo sottocampo proprio, e quindi, se di caratteristica due, il polinomio $x^{2}-b x-c$ è separabile su $F$, il grupp̀o degli automorfismi del piano di Hall $S(F, b, c)$ è isomorfo al gruppo delle collineazioni dello $S_{4}$ che mantengono fisso lo $S_{3}$ e inducono ivi una collineazione rispetto alla quale la congruenza di rette $H(F, b, c)$, che individua il piano $S(F, b, c)$, è unita.

Questi risultati, conseguiti nel presente lavoro, sono già stati parzialmente enunciati, senza dimostrazione, in [6] ( $\left.{ }^{6}\right)$. Vi $\partial$ un miglioramento dei teoremi, enunciati in [6], poichè essi sono estesi al caso di campi di caratteristica due che siano perfetti; ma $\mathrm{i}$ campi $F$, considerati in questo lavoro, si suppongono non isomorfi a un loro sottocampo proprio.

Oltre a ciò si dimostra, n. 4 , che $i l$ piano di Hall $S(F, b, c)$, se $F$ non $\grave{e}$ isomorfo al campo di Galois di ordine tre, non è un piano sopra un quasicorpo associativo.

1. Si consideri un corpo $K$ (non necessariamente commutativo) e lo spazio lineare affine (7) a $2 n(n \geq 2)$ dimensioni $S_{2 n}$ sopra $K$ : sia $S_{2 n-1}^{\infty}$ lo spazio improprio di $S_{2 n}$. Una congruenza di spazi $(n-1)$-dimensionali in $S_{2 n-1}^{\infty}$ è un insieme $C_{n-1}$ di sottospazi a $n-1$ dimensioni di $S_{2 n-1}^{\infty}$, tale che ogni punto di $S_{2 n-1}^{\infty}$ appartenga a uno e a un solo elemento di $C_{n-1}$. E noto che la con. gruenza $C_{n-1}$ individua un piano di traslazione $S\left(K, C_{n-1}\right)$ i cui punti sono i punti (propri) dello $S_{2 n}$ e le cui rette sono quei sottospazi di dimensione $n$ di $S_{2 n}$ che hanno in comune con $S_{2 n-1}^{\infty}$ un elemento di $C_{n-1}$ : diremo $S\left(K, C_{n-1}\right)$ piano di traslazione relativo al corpo $K$ e alla congruenza $C_{n-1}$.

Se, ora, si considerano due piani di traslazione $S\left(K, C_{n-1}\right)$ e $S\left(K, C_{n-1}^{\prime}\right)$ relativi al corpo $K$ e alle congruenze $O_{n-1}$ e $C_{n-1}^{\prime}$ rispettivamente, un isomor-

(5) Intendendo con clò che deve esistere una collineazione di $S_{3}$, inerente un automorfismo $\varphi$ di $F$, la quale trasforma le rette appartenenti a $\bar{H}(F, b, c)$ in quelle appartenenti a $\bar{H}\left(F, b^{\prime}, c^{\prime}\right)$.

(5) E, sempre parzialmente, citati in [4].

(7) Destro o sinistro. Nel seguito, per comodità di linguaggio, ci riferiremo allo spazio affine ampliato con $i$ suoi elementi impropri : specificheremo, quando eiò non risulterà chiaro dal contesto, se gli elementi che si considerano sono elementi dello spazio affine o dello spazio affine ampliato. Per le nozioni di geometria proiettiva, delle quali si fa uso, si rimanda al trattato [9]. 
fismo affine tra $i$ due piani è una corrispondenza biunivoca tra i punti propri di $S_{2 n}$, che muta punti appartenenti a una retta di $S\left(K, C_{n-1}\right)$ in panti appartenenti a una retta di $S\left(K, C_{n-1}^{\prime}\right)$, e viceversa; di conseguenza ([7], pp. 11-12), un isomorfismo affine tra $S\left(K, C_{n-1}\right)$ e $S\left(K, O_{n-1}^{\prime}\right)$ pone una corrispondenza biunivoca tra gli elementi delle congruenze $C_{n-1}$ e $C_{n-1}^{\prime}$.

Cio premesso, dimostriamo il seguente

LeMMA I. - Se un isomorfismo affine ? tra $i$ piani $S\left(K, C_{n-1}\right)$ e $S\left(K, C_{n-1}^{\prime}\right)$ trasforma $i$ punti di una particolare retta $r$ di $S_{2 n}$ in punti di una retta $r^{\prime}$, allora $\vartheta$ muta terne di punti allineati $d i S_{2 n}^{-}$in terne di punti che verificano la medesima condizione.

Sia, infatti, $s$ una retta di $S_{2 n}$ incidente $r$ ma sghemba con l'elemento di $C_{n-1}$ passante per il punto $R$ improprio di $r$ siano $A_{i}(i=1,2,3)$ tre suoi punti $\theta S$ il suo punto improprio: cominciamo col provare che $\vartheta$ trasforma i tre punti $A_{i}$ in tre punti $A_{i}^{\prime}=\vartheta\left(A_{i}\right)$ che risultano allineati e, quindi, trasforma i punti della retta $s$ in punti di una retta $s^{\prime}$. Perciò si consideri un punto $T$ della retta congiungente $R$ e $S(T \neq R, T \neq S)$ e sia $S_{n-1}(T)$ l'elemento di $C_{n-1}$ passante per il punto $T ; S_{n-1}(T)$ è congiunto con le rette $s$ e $r$ da un sottospazio di dimensione $n+1$ dello $S_{2 n}$. I tre spazi $S_{n}^{i}$ che proiettano i tre punti $A_{i}$ da $S_{n-1}(T)$ intersecano $r$ in tre punti $B_{i}$ e sono mutati da $q$ in tre spazi a $n$ dimensioni di $S_{2 n}, \vartheta\left(S_{n}^{i}\right)=S_{n}^{i}$, risultando $\left.\vartheta_{[}^{\prime} S_{n-1}(T)\right]=S_{n-1}^{\prime \prime}(T) \subset S_{n}^{\prime i}$ e $\vartheta\left(B_{i}\right)=B_{i}^{\prime}, A_{i}^{\prime} \subset S_{n}^{\prime i}$. Essendo i punti $B_{i}^{\prime}$ allineati (per ipotesi) gli spazi $S_{n}^{\prime i}$ appartengono a un sottospazio $(n+1)$-dimensionale di $S_{2 n}$, il quale interseca lo $S_{n}$ di $S_{2 n}$ rappresentante la retta di $S\left(K, C_{n-1}^{\prime}\right)$ individuata dai tre punti $A_{i}^{\prime}$ [i quali in $S\left(K, C_{n-1}^{\prime}\right)$ sono dipendenti] in una retta (e per constatare ciò basta riferirsi agli elementi impropri di tali spazi) che contiene i punti $A_{i}$. E cosi provato che i punti di una qualunque retta di $S_{2 n}$ che incida la retta $r$, ma non l'elemento di $C_{n-1}$ passante per il punto improprio di $r$, sono mutati dall'isomorfismo affine $\vartheta$ in punti appartenenti a una retta dello spazio $S_{2 n}$. Se, poi, 3 è una retta di $S_{2 n}$ sghemba con $r$ e con l'elemento $S_{n-1}(R)$ di $C_{n-1}$ passante per $R$, si verifica facilmente (sempre riferendosi agli elementi inpropri degli spazi che si considerano) l'esistenza di una retta, analoga alla retta $s$, incidente $r$ e $s$ : da cio si deduce la tesi del lemma per la retta $s$. Giunti a questo punto è d'immediata verifica che anche i punti di una retta di $S_{2 n}$ incidente $S_{n-1}(R)$ sono mutati da $\vartheta$ in punti appartenenti a una retta di $S_{2 n}$.

Osservazione. - Si noti che nel precedente lemma I l'ipotesi che l'isomorfismo $\vartheta$ muti i punti di una retta $r$ di $S_{2 n}$ in punti di una retta $r^{\prime}$ non è sovrabbondante. Se, infatti, il corpo $K$ è isomorfo al campo reale $R F$, si consideri nello $S_{3}^{\infty}$ improprio dello $S_{4}$ lineare affine sopra $R F$ una congruenza lineare ellittica $C_{1}$ di rette: il piano di traslazione $S\left(R F, C_{1}\right)$ relativo al campo $R F$ e alla congruenza $C_{1}$ \& isomorfo al piano lineare affine sopra il campo 
dei numeri complessi $C F$. Ciò significa che si può fissare in $S\left(R F, C_{1}\right)$ un riferimento il quale permette di' introdurvi delle cocrdinate estratte dal campo dei numeri complessi. Detto $\vartheta$ un automorfismo del campo $O F$ che nón coincida con l'automorfismo identico o col coniugio, e l'esistenza di un tale auto. morfismo \& provata in [9], sia $\Phi$ una collineazione dello $S\left(R F, C_{2}\right)$, ampliato con i suoi elementi impropri, la quale mantenga fissa la retta impropria dello $S\left(R F, C_{1}\right)$ e sia inerente all'automorfismo $\vartheta$. Ci si rende facilmente conto che, nello $S_{4}$, la collineazione $\Phi\left({ }^{8}\right)$ rappresenta una trasformazione che non conserva le condizioni di appartenenza.

2. - Por stabilire un secondo lemma, necessario per il seguito, inerente al piano di traslazione $S\left(K, C_{n-1}\right)$ relativo al corpo $K$ e alla congruenza $C_{n-1}$, è opportuno richiamare alcuni concetti essenziali e introdurre alcune locuzioni. Perciò cominciamo col dire punto improprio dello $S\left(K, C_{n-1}\right)$ un elemento dell'insieme quoziente della totalità delle rette di $S\left(K, C_{n-1}\right)$ rispetto alla nozione di parallelismo e con l'identificare i punti impropri di $S\left(K, C_{n-1}\right)$ con gli $S_{n-1}$ di $S_{2 n-1}^{\infty}$ appartenenti alla congruenza $C_{n-1}$. Ciò premesso, siano 0 , $E, D, \nabla$ quattro punti, a tre a tre indipendenti, di $S\left(K, C_{n-1}\right)$ e i punti $U$ e $V$ siano impropri; sono individuati i tre $S_{n}$ di $S_{2 n}$ rappresentanti le rette di $S\left(K, C_{n-1}\right)$ congiungenti il punto $O$ con i punti $E, U, V$ : diciamoli $O E, x, y$ rispettivamente $\mathrm{e}$ siano $e^{\infty}, x^{\infty}, y^{\infty}$ gli elementi di $C_{n-1}$ appartenenti a $O E$, a $x$, a $y$.

Si è così fissato un riferimento affine $R$ in $S\left(K, C_{n-1}\right)$ e ad ogni punto di tale piano rimane associata una coppia ordinata di punti di $y$ mediante l'operazione ternaria di M. HALL: tra i punti di $y$ si introduce una struttura algebrica rispetto alla quale essi costituiscono un quasicorpo $Y$. Detto $E_{y}$ il punto proiezione di $E$ da $x^{\infty}$ su $y$, vale il seguente

LEMMA II: Gli elementi del quasicorpo $Y$ che appartengono alla retta di $S_{2 n}$ congiungente $i$ punti $O$ e $E_{y}$ costituiscono un sottoquasicorpo di $Y$ : tale sottoquasicorpo è isomorfo al corpo $K$.

Detta $r_{y}$ la retta di $S_{2 n}$ conginngente $O$ e $E_{y}$, sia $r_{e}$ la retta dj $S_{2 n}$ proiezione di $r_{y}$ da $x^{\infty}$ sullo $S_{n} O E$ di $S_{2 n}$ e $r_{x}$ la retta di $S_{2 n}$ proiezione di $r_{e}$ da $y^{\infty}$ sullo $S_{n} x$ di $S_{2 n}$. Le tre rette $r_{y}, r_{e}, r_{x}$ appartengono al piano $\pi$ comune ai due $S_{n+1}$ congiungenti, rispettivamente, la retta $r_{y}$ con $x^{\infty}$ e la retta $r_{e}$ con $y^{\infty}$; a tale piano appartengono, quindi, anche i punti $E$ e $E_{y}$. Per dimostrare il lemma II basta osservare che per operare sui punti di $r_{y}$ mediante l'operazione ternaria di M. HALL, allo scopo di definire la struttura algebrica ad essi spettante come elementi di $Y$, è equivalente ad operare su $\pi$ (che, come sot-

(8) Pur agendo in modo da porre una corrispondenza biunivoca tra i punti di $S_{4}$, biunivoca tra gli elementi della congruenza $C_{4}$, e trasformando i punti appartenenti a un piano per una retta di $C_{1}$ in punti ohe verificano la stessa condizione. 
tospazio di $S_{2 n}$, ̀̀ un piano affine irriducibile) in relazione al riferimento $O^{\prime}$, $E^{\prime}, U^{\prime}, V^{\prime}$ ove $O^{\prime}=0, E^{\prime}=E, U^{\prime}=\pi \cap x^{\infty}, V^{\prime}=\pi \cap y^{\infty}$. Ma, poichè in $\pi$ vale universalmente il teorema di Desarauns, segue che $i$ punti di $r_{y}$ costituiscono un sottocorpo di $Y$ : tale sottocorpo è isomorfo a $K$ poichè $\pi$ è un piano di $S_{2 n}$.

3. - In questo numero, e nei successivi, ci occuperemo di piani di traslazione relativi a particolari corpi $K$ e particolari congruenze $C_{n-1}$. Specia. lizzeremo il corpo $K$ in un campo (corpo commutativo) $F$, non isomorfo al campo di GaLOIS GF (2), tale che esista un polinomio $x^{2}-b x-c$ con coefficienti in $F$ e irriducibile su $F$, e specializzeremo la congruenza $C_{n-1}$ in una congruenza $C_{1}=H(\mathrm{~F}, \mathrm{~b}, \mathrm{c})$ di rette (appartenente allo $S_{3}^{\infty}$ improprio dello $S_{4}$ lineare affine sopra il campo $F$ ) costituita dalle rette dello $S_{3}^{\infty}$ rappresenta. bili in una delle seguenti forme $\left({ }^{8}\right)$ :

$$
\begin{aligned}
& \alpha) \equiv\left\{\begin{array}{l}
y_{1}=a_{1} x_{1}-\left(a_{1}^{2}-b a_{1}-c\right) a_{2}^{-1} x_{2} \\
y_{2}=a_{2} x_{1}+\left(b-a_{1}\right) x_{2}
\end{array}\right. \\
& \left.\alpha^{\prime}\right) \equiv y_{1}-a_{1} x_{1}=y_{2}-a_{1} x_{2}=0 \\
& \beta) \equiv x_{1}=x_{2}=0
\end{aligned}
$$

ove $a_{1}, a_{2}\left(a_{2} \neq 0\right)$ sono elementi del campo $F$.

Il piano di traslazione retativo al campo $F$ e alla congruenza $H(F, b, c)$, il quale, nel seguito, si indichera con $i S(F, b, c)$ e si dirà piano di HaLL, si può anche definire [5] come piano di traslazione sopra il quasicorpo di HaLL $J(F, b, c)$; tale quasicorpo e costituito dalle coppie ordinate $\left(x_{1}, x_{2}\right)$ di elementi estratti da $F$, quando le operazioni di somma e prodotto tra coppie siano definite alla seguente maniera:

definizione di somma

$$
\left(x_{1}, x_{2}\right)+\left(z_{1}, z_{2}\right)=\left(x_{1}+z_{1}, x_{2}+z_{2}\right)
$$

definizione di prodotto

$$
\left(x_{1}, x_{2}\right)\left(a_{1}, a_{2}\right)=\left(y_{1}, y_{2}\right)
$$

$\left(y_{1}, y_{2}\right)$ ottenendosi mediante le $\left.\left.\alpha\right) \circ \alpha^{\prime}\right)$ a seconda che $a_{2}$ sia diverso o uguale a zero.

La congruenza $H(F, b, c)$ contiene il regolo della quadrica $x_{1} y_{2}-x_{2} y_{1}=0$ costituito dalle rette $\alpha^{\prime}$ ) e $\beta$ ); l' ulteriore regolo della quadrica esaurisce, insieme alle rette $\alpha$ ), la congruenza lineare $\bar{H}(F, b, c)$ di rette di $\mathcal{S}_{3}^{\infty}$ che si

( $\left.{ }^{9}\right) x_{1}, x_{2}, y_{1}, y_{2}$ sono coordinate proiettive omogenee in $S_{3}^{\infty}$, e, quindi, coordinate affini dello $S_{4}$. 
appoggiano alle due rette (dello spazio tridimensionale lineare sopra il campo $F^{\prime}$ estensione algebrica di $F$ mediante il polinomio $\left.x^{2}-b x-c\right)$ di rispettive equazioni

$y_{1}-k_{1} x_{1}=y_{2}-k_{1} x_{2}=0, \quad y_{1}-k_{2} x_{1}=y_{2}-k_{2} x_{2}=0 \quad\left[k_{i}^{2}-b k_{i}-c=0 ; i=1,2\right]$.

Le precedenti rette risultano distinte se $k_{1} \neq k_{2}$ : tale eventualità non si presenta di necessità se il campo $F$, avendo caratteristica due, non è perfetto.

Il piano $\bar{S}(F, b, c)$ ralativo al campo $F$ e alla congruenza lineare $\bar{H}(F, b, c)$ è desarguesiano; infatti vale la proposizione:

Il piano $\bar{S}(F, b, c)$ è isomorfo al piano affine lineare sopra il campo estensione algebrica di $F$ mediante il polinomio $x^{2}-b c^{-1} x-c^{-1}(e$, quindi, al piano affine lineare sopra il campo $F^{\prime}$ ).

Per provarlo si osservi che l'affinità di $S_{4}$ di equazioni

$$
x_{1}^{\prime}=x_{1} \quad x_{2}^{\prime}=y_{1} \quad y_{1}^{\prime}=x_{2} \quad y_{2}^{\prime}=y_{2}
$$

trasforma la congruenza lineare $\bar{H}(F, b, c)$ in una congruenza lineare $\bar{H}^{\prime}(F, b, c)$ costituita dalle rette rappresentabili, in $S_{3}^{\infty}$, sotto la forma (si trascurano gli apici relativi alle variabili):

$$
\begin{aligned}
& \bar{\alpha})=\left\{\begin{array}{l}
y_{1}=a_{1} x_{1}+c^{-1} a_{2} x_{2} \\
y_{2}=a_{2} x_{1}+\left(c^{-1} b a_{2}+a_{1}\right) x_{2}
\end{array}\right. \\
& \bar{\beta})=x_{1}=x_{2}=0
\end{aligned}
$$

Il piano di traslazione $\overline{S^{\prime}}(F, b, c)$ relativo al campo $F$ e alla congruenza $\bar{H}^{\prime}(F, b, c)$ $\otimes$ isomorfo a quello relativo al campo $F$ e alla congruenza $\bar{H}(F, b, c)$, poichè la precedente affinità pone un isomorfismo affine tra i due piani. Se in $\overline{S^{\prime}}(F, b, c)$ si fissa un riférimento affine nel quale gli assi $x, y$ e la retta unita siano i piani di $S_{4}$ di rispettive equazioni $y_{1}=y_{2}=0, x_{1}=x_{2}=0, y_{1}-x_{1}=y_{2}-x_{2}=0$ ed il punto unità sia il punto $E=(1,0,1,0)$, si genera, mediante l'operazione ternaria di M. HALL, una struttura algebrica tra i punti dell'asse $y$, rispetto alla quale l'insieme di tali punti è un quasicorpo. Si verifica che quella struttura algebrica è definita da:

$$
\begin{array}{ll}
\text { somma } & \left(0,0, x_{1}, x_{2}\right)+\left(0,0, a_{1}, a_{2}\right)=\left(0,0, x_{1}+a_{1}, x_{2}+a_{2}\right) \\
\text { prodotto } & \left(0,0, x_{1}, x_{2}\right)\left(0,0, a_{1}, a_{2}\right)=\left(0,0, y_{1}, y_{2}\right)
\end{array}
$$

ove $y_{1}, y_{2}$ si ottengono da $x_{1}, x_{2}, a_{1} a_{2}$ mediante le uguaglianze $\left.\bar{\alpha}\right)$. Risulta:

$$
\begin{gathered}
\left(0,0, a_{1}, a_{2}\right)=\left(0,0, a_{1}, 0\right)+\left(0,0, a_{2}, 0\right)(0,0,0,1) \text { e }(0,0,0,1)^{2}= \\
=\left(0,0, c^{-1}, c^{-1} b\right)=\left(0,0, c^{-1} b, 0\right)(0,0,0,1)+\left(0,0, c^{-1}, 0\right) .
\end{gathered}
$$

Poichè gli elementi dell'asse $y$ del tipo $\left(0,0, y_{1}, 0\right)$ costituiscono, rispetto alla somma e al prodotto già definiti, un campo isomorfo al campo $F$, pos- 
siamo affermare che l'elemento $(0,0,0,1)$ verifica l'equazione $x^{2}-c^{-1} b x-c^{-1}=0$ con coefficienti in $F$, e da cio segue la proposizione enunciata.

Concludendo, si ha che:

Ogni congruenza $H(F, b, c)$, inerente un piano di $M$. Hall, individua una congruenza $\bar{H}(F, b, c)$ lineare di rette di $S_{3}^{\infty}$; il piano di traslazione $\bar{S}(F, b, c)$ relativo al campo $\dot{F}$ e alla congruenza $\bar{H}(F, b, c)$ è isomorfo al piano affine lineare sopra il campo estensione algebrica di $F$ mediante il polinomio $x^{2}-b x-c$.

4. - Si consideri, ancora, il piano di HaLI $S(F, b, c)$ e, nello $S_{s}^{\infty}$, la congruenza $H(F, b, c)$ ad esso associata. Il gruppo $G$ delle omografie di $S_{3}^{\infty}$ ohe sono rappresentabili con equazioni del tipo:

$$
G \equiv\left\{\begin{array}{l}
\rho x_{1}=\sigma\left(\gamma x_{1}{ }^{\prime}+\delta x_{2}{ }^{\prime}\right)+\tau\left(\gamma y_{1}{ }^{\prime}+\delta y_{2}{ }^{\prime}\right) \\
\rho x_{2}=\sigma\left(\mu x_{1}^{\prime}+v x_{2}{ }^{\prime}\right)+\tau\left(\mu y_{1}{ }^{\prime}+\nu y_{2}^{\prime}\right) \\
\rho y_{1}=\sigma \tau\left(\gamma x_{1}{ }^{\prime}+\delta x_{2}\right)+(\sigma+b \tau)\left(\gamma y_{1}^{\prime}+\delta y_{2}^{\prime}\right) \\
\rho y_{2}=\tau \tau\left(\mu x_{1}^{\prime}+\gamma x_{2}^{\prime}\right)+(\sigma+b \tau)\left(\mu y_{1}^{\prime}+\gamma y_{2}^{\prime}\right)
\end{array}\right.
$$

(ove $\gamma, \delta, \mu, \gamma, \sigma, \tau$ sono dei parametri omegenei scelti in $F$ con la condizione che $\gamma \nu-\delta \mu \neq 0, \sigma, \tau$ non siano contemporaneamente nulli e $\rho$ è un elemento di $F$ diverso dallo zero) trasforma la congruenza $H(F, b, c)$ in sè mantenendo fisso l'insieme delle sue rette appartenenti alla quadrica $x_{1} y_{2}-x_{2} y_{1}=0$.

Si verifica facilmente che le rette $\alpha^{\prime}$ ) e $\beta$ ), le quali costituiscono un regolo della precedente quadrica, appartengono a un insieme di transitivita per $G$ : cio discende dall'osservare che le uniche rette di $x_{1} y_{2}-x_{2} y_{1}=0$ che sono mantenute fisse da $G$ appartengono allo spazio, a tre dimensioni, lineare sopra il campo $F^{\prime}$, ove $F^{\prime}$ indica, al solito, il campo estensione algebrica di $F$ mediante il polinomio $x^{2}-b x-c$, e coincidono con gli assi della congru. enza lineare $\bar{H}(F, b c)$ associata (cfr. n. 3) alla congruenza $H(F, b, c)$.

Inoltre, se si considerano le rette $\alpha$ ) della congruenza $H(F, b, c)$ e le trasformazioni di $G$ ottenute per $\delta=\mu=\tau=0$, si vede immediatamente che tali trasformazioni costituiscono un sottogruppo di $G$ che trasforma la retta di a) individuata dagli elementi $a_{1}, a_{2}\left(a_{2} \neq 0\right)$ di $F$ nella retta dell' insieme $\alpha$ ) individuata dagli elementi $a_{1}^{\prime}=a_{1} \otimes a_{2}^{\prime}=a_{2} \gamma \gamma^{-1}$ di $F$.

Le precedenti considerazioni ci permettono di affermare: Nessuna retta di $H(F, b, c)$ è mutata in sè da ogni trasformazione di $G$.

Ciò premesso, possiamo provare che:

In un piano di Hall esiste una e una sola retta $r$ rispetto alla quale esso risulti $(r-r)$-transitivo $\left({ }^{10}\right)$ (tale retta coincidendo con la retta impropria del piano).

(10) Un piano di traslazione $S$ si dice $(r-r)$ - transitivo se esiste, in $S$, un gruppo di omologie speciali aventi per asse la retta $r$ transitivo sui punti di $S$ non appartenenti ad $r$; in tal caso si dice anche che il piano $S$ è microdesarguesiano rispetto alla retta $r$. 
Ricordato che un piano di traslazione è, per definizione, microdesarguesiano rispetto alla retta impropria, sia $r$ un'eventuale retta di $S(F, b, c)$, distinta dalla retta impropria, rispetto alla quale il piano risulti $(r-r)$-transitivo. Detta $h$ la retta di $H(F, b, c)$ immagine del punto improprio di $r$, si osservi che nell' ipotesi ammessa, come è ben noto $[3], S(F, b, c)$ dovrebbe risultare $(s-s)$-transitivo rispetto ad ogni sua retta $s$ avente punto improprio $h$. Ma il gruppo delle affinità di $S_{4}$ che inducono nello $S_{3}^{\infty}$ improprio di $S_{4}$ una trasformazione di $G$, costituisce un gruppo di automorfismi affini di $S(F, b, c)$; per un'osservazione precedente esiste almeno uno di tali antomorfismi affini che muta la retta $h$ in una retta $h^{\prime} \in H(F, b, c)$ risultando $h^{\prime} \neq h$. Poichè un automorfismo affine $\vartheta$ di un piano grafico irriducibile conserva $i$ gruppi di omologie spettanti al piano, (il che significa, ad esempio, che se il piano risulta $(r-r)$-transitivo e $r^{\prime}=\vartheta(r)$ allora il piano deve essere anche $\left(r^{\prime}-r^{\prime}\right)$-tran. sitivo) il piano di HaLL $S(F, b, c)$, oltre ad essere $(s-s)$-transitivo per ogni sua retta $s$ avente come punto improprio $h$, dovrebbe risultare $\left(s^{\prime}-s^{\prime}\right)$-transitivo anche per ogni sua retta $s^{\prime}$ passante per il punto improprio $h^{\prime}$. Quindi il piano $S(F, b, c)$ risulterebbe microdesarguesiano [3]: esso, cioè, sarebbe $(r-r)$-transitivo rispetto ad ogni sua retta $r$. Ciò è assurdo, poichè in tal caso il piano $H(F, b, c)$ dovrebbe essere un piano di $H$. Moufang ([7], pp. 186-198) e tra i sistemi algebrici da esso individuati mediante l'operazione ternaria di M. HALL, in relazione ad un qualsiasi riferimento, non ve ne sarebbe alcuno isomorfo al quasicorpo di HALL $J(F, b, c)$.

Ricordato ohe si dice isomorfismo tra due piani di traslazione una corrispondenza biunivoca tra i loro punti la quale conserva gli allineamenti, in base al risultato precedente possiamo affermare che:

Ogni isomorfismo tra due piani di Hall $S(F, b, c)$ e $S\left(F, b^{\prime}, c^{\prime}\right)$, relativi al campo $F$ e alle congruenze $H(F, b, c)$ e $H\left(F, b^{\prime}, c^{\prime}\right)$ rispettivamente, trasforma punti impropri in punti impropri: esso, cioè, risulta un isomorfismo affine.

Si noti che il precedente teorema è conosciuto in generale per piani di traslazione (propri) qualsiansi (cfr. [7], p. 207, per piani di traslazione di caratteristica diversa da due; [8] nol caso di piani di traslazione di caratteristica due). Ho ritrovato il risultato per $i$ piani di HaLL poichè, in questo caso, si giunge ad esso con considerazioni molto semplici mettendo, inoltre, in evidenza il grappo $G$ e, quindi, un gruppo di automorfismi del piano di HALL $S(F, b, c)$. L'opportunita delle considerazioni svolte in questo numero è confermata anche dal fatto che, mediante esse, si perviene al seguente risultato:

Ogni piano di Hall $S(F, b, c)$, nel caso che. $F$ non sia isomorfo al campo di Galois di ordine tre, non è un piano associativo; ossia: in $S(F, b, c)$ non esistono riferimenti che, mediante l'operazione ternaria $d i M$. Hall, generino un quasicorpo associativo.

Infatti, dalle considerazioni svolte all'inizio del presente numero discende facilmente che $G$ opera su $H(F, b, c)$ in modo tale che nessuna copia di rette 
di $H(F, b, c)$ è unita per ogni trasformazione del gruppo. Esiște, quindi, in $S(F, b, c)$ un gruppo di automorfismi che non muta in sè una coppia di punti impropri del piano; l'esistenza di un riferimento in $S(F, b, c)$ che generi un quasicorpo associativo (necessariamente non distributivo) $\dot{e}$, quaindi, assurda ([7], p. 218, teorema 17 ) se il campo $F$ non ha ordine tre.

5. - I risultati conseguiti nei numeri 3 e 4 ei permettono di affrontare il problema, posto nell'introduzione del presente lavoro, della caratterizzazione dei quasicorpi di HaLL geometricamente isomorfi o, se si vuole, dei piani di HALI che risultano tra loro isomorfi. Per procedere più speditamente nel seguito è opportuno premettere la seguente proposizione:

$I$ sottocampi del quasicorpo di Hall $J(F, b, c)$ che risultano isomorfi al campo $F$ sono contenuti nel sottoinsieme di $J(F, b, c)$ costituito dai suoi elementi del tipo $\left(x_{1}, 0\right)$.

Poichè ogni sottocampo $S C$ di $d(F, b, c)$ deve contenere l'elemento $(1,0)$ se $x=\left(x_{1}, x_{2}\right) \in S C$ allora anche $x+1=\left(x_{1}+1, x_{2}\right) \in S C$; da cio segue, quando la caratteristica di $F$ è diversa da due, che gli elementi di $S C$ sono tutti del tipo $\left(x_{1}, 0\right)$, poichè, in tal caso, $x(x+1) \neq x^{2}+x$ se $x_{2} \neq 0$.

Si consideri, ora, il caso in cui $F$ abbia caratteristica due. L'uguaglianza $x x=x x^{2}$ implica $0 x_{2}=0$ oppure $b^{2} b+1 \mid x_{1}=b^{2} c+c^{2}+b c+c$; l'ultima equazione in $x_{1}$, per un'osservazione precedente, deve avere due soluzioni distinte, il che comporta $b=c=1$. Rimane, quindi, da discutere solamente il caso di quasicospi di HALL $J(F, 1,1)$ relativi a un campo $F$ di caratteristica due. Poichè l'equazione $x^{2}-x-1=0$ è risolubile nel campo di Galors di ordine quattro, si può supporre che $F$ abbia più di quattro elementi; in tal caso, in $S C$, oltre a $x$ e $x+1$ non può essere contenuto $y=\left(y_{1}, y_{2}\right)$ e distinto da $x$ e $x+1$ se $x_{2} y_{2} \neq 0$. Infatti, se $x_{2}+y_{2} \neq 0$ risulta $(x+y)^{2} \neq x^{2}+y^{2}$ mentre, se $x_{2}=y_{2}, x y=y x$ comporta $y_{1}=x_{1} \circ y_{1}=x_{1}+1$. Cio significa che, nel caso in discussione, un sottocampo $S C$ di $J(F, 1,1)$ che sia isomorfo a $F$ o necessariamente costituito da elementi del tipo $\left(x_{1}, 0\right)$ di $J(F, 1,1)$ fatta, al più, eccezione per due elementi $x, x+1$ che non risultano di tale tipo. Ma in $S C$ vi è, quindi, un elemento $z=\left(z_{1}, 0\right)$ con $z_{1} \neq 0,1$ e, quindi, accanto a $x$ e $x+1$ dovrebbe esserci anche l'elemento $x z=\left(x_{1} z_{1}, x_{2} z_{1}\right)$ ove $x_{2} z_{1} \neq x_{2}$ : \& assurdo, cioè, supporre $x \in S C$, se $x_{2} \neq 0$, poichè $x z \in S C$ è in contraddizione con quanto già stabilito.

Ciò premesso, possiamo dimostrare il seguente

Teorema I. - Siano $S(F, b, c)$ e $S\left(F, b^{\prime}, c^{\prime}\right)$ due piani di Hall relativi al campo $F$ che si suppone dotato di almeno quattro elementi, non isomorfo ad un suo sottocampo proprio e, quindi, necessariamente perfetto. Siano, inoltre, $H(F, b, c)$ e $H\left(F, b^{\prime}, c^{\prime}\right)$ le congruenze di rette relative a $S(F, b, c)$ e a $S\left(F, \cdot b^{\prime}, c^{\prime}\right)$ rispettivamente e $\bar{H}(F, b, c)$ e $\bar{H}\left(F, b^{\prime}, c^{\prime}\right)$ le congruenze lineari ellettiche di rette ad esse associate. Condizione necessaria e sufficiente perchè $i$ piani $S(F, b, c)$ 
e $S\left(F, b^{\prime}, c^{\prime}\right)$ risultino isomorfi è che esista una collineazione dello spazio tridimensionale lineare sopra il campo $F$ che trasformi la congruenza $\bar{H}(F, b, c)$ nella congruenza $\bar{H}\left(F, b^{\prime}, c^{\prime}\right)$.

Per dimostrare la sufficienza della condizione si osservi che, se esiste una collineazione $\bar{\Phi}$ dello $S_{3}^{\infty}$ improprio dello $S_{4}$ lineare affine sopra il campo $F$ che trasforma la congruenza $\bar{H}(F, b, c)$ nella congruenza $\bar{H}\left(F, b, c^{\prime}\right)$ deve esistere una collineazione $\Phi$ di $S_{3}^{\infty}$ la quale, oltre a trasformare la congruenza $\bar{H}(F, b, c)$ nella congruenza $\bar{H}\left(F, b^{\prime}, c^{\prime}\right)$ mantiene fissa la quadrica $Q=x_{2} y_{2}$ $-x_{2} y_{1}=0$ di $S_{3}^{\infty}$. Infatti la $\bar{\Phi}$ muta la $Q$, un cui regolo appartiene a $\bar{H}(F, b, c)$, in una quadrica $Q^{\prime}$ che ha un regolo contenuto in $\bar{H}\left(F, b^{\prime} c^{\prime}\right)$; le quadriche $Q$ e $Q^{\prime}$, se sono distinte, individuano un fascio di quadriche. Si verifica facilmente che, nelle ipotesi poste, il gruppo delle omografie dello $S_{3}^{\infty}$, che mantiene fisso tale fascio e la congruenza $\bar{H}\left(F, b^{\prime}, c^{\prime}\right)$, è un gruppo transitivo sulle quadriche non degeneri del fascio. Ma, allora, $\Phi$ trasforma la congruenza $H(F, b, c)$ nella congruenza $H\left(F, b,^{\prime} c^{\prime}\right)$ e, quindi, ogni affinità dello $S_{4}$, inerente un opportuno automorfismo di $F$, che induca nello $S_{3}^{\infty}$ la collineazione $\Phi$, pone un isomorfismo affine tra i piani $S(F, b, c)$ e $S\left(F, b^{\prime}, c^{\prime}\right)$.

Dimostriamo, ora, che la condizione è necessaria. Raggiungeremo lo scopo provando che un eventuale isomorfisino tra i piani $S\left(F^{\prime}, b, c\right)$ e $S\left(F, b^{\prime}, c^{\prime}\right)$ induce, in $S_{3}^{\infty}$, una collineazione $\bar{\Phi}$ che trasforma la congruenza $\bar{H}(F, b, c)$ nella congruenza $\left.\vec{H}_{(} F^{\prime}, b^{\prime}, c^{\prime}\right)$. Percio si consideri un riferimento $R$ in $S\left(l^{\prime}, b, c\right)$ che generi, mediante l'operazione ternaria di M. HALL, un quasicorpo isomorfo al quasicorpo $J(F, b, c)$ : siano, in relazione al riferimento $R, O$ l'origine, $E$ il punto unità, $U$ e $V$ i punti impropri degli assi $x$ ed $y$ rispettivamente. Detto w un eventuale isomorfismo tra $S(F, b, c)$ e $S\left(F, b^{\prime}, c^{\prime}\right)$ siano $O^{\prime}=\vartheta(O), E^{\prime}=\vartheta(E)$, $U^{\prime}=\vartheta(U) . V^{\prime}=\vartheta(V)$ i quattro punti trasformati, mediante l'isomorfismo $\$$, dei punti che individuano il riferimento $R$ in $S(F, b, c)$ : il riferimento $K^{\prime}$, individuato in $S\left(F, b^{\prime}, c^{\prime}\right)$ dai quattro punti $O^{\prime}, E^{\prime}, U^{\prime}, V^{\prime}$ genera un quasicorpo isomorfo a $J(F, b, c)$. Inoltre l'isomorfismo $\vartheta$ è necessariamente affine (cfr. 4): ciò significa che i punti $O^{\prime}$ e $E^{\prime}$ sono propri. Per il lemma II i punti della retta $O E_{y}\left[O^{\prime} E_{y^{\prime}}^{\prime}\right]\left(E_{y}\right.$ indica, al solito, il punto proiezione di $E$ da $U$ sulla retta $O V$ ) costituiscono un sottocorpo, isomorfo al campo $F$, del quasicorpo $J(F, b, c)$ generato dal riferimento $O, E, U, V$ di $S(F, b, c)\left[O^{\prime}, E^{\prime}, U^{\prime}, V^{\prime}\right.$ di $\left.S\left(F, b, c^{\prime}\right)\right]$. Tenendo conto della proposizione enunciata all'inizio del presente numero, si puo affermare che il quasicorpo di HaLL $J(F, b, c)$ ammette, nelle ipotesi poste, un unico sottocorpo isomorfo al campo $F$ e che, quindi, $\vartheta$ trasforma i punti della retta $O E_{y}$ di $S_{4}$ nei punti della retta $O^{\prime} E_{y^{\prime}}^{\prime}$ ponendo tra le due rette una corrispondenza biunivoca. Ma allora il lemma $I$, applicato all'isomorfismo $\vartheta$ e all'isomorfismo $\vartheta^{-1}$, ci assicura che $\vartheta$ trasforma terne di punti allineati in $S_{4}$ in terne di punti che verificano la medesima condizione, e viceversa. Inoltre $\vartheta$ agisce nello $S_{3}^{\infty}$ mutando rette di $H(F, b, c)$ in rette di $H\left(F, b^{\prime}, c^{\prime}\right)$ : la corrispondenza tra quelle rette di $S_{3}^{\infty}$ può essere estesa ai punti di tale spazio 
associando ad ogni punto $P$ di $S_{3}^{\circ}$ il punto improprio delle rette di $S_{4}$ trasformate, mediante $\vartheta$, delle rette di $S_{4}$ passanti per $P$. In tal modo si è posta nello $S_{3}^{\infty}$ una corrispondenza biunivoca tra i punti la quale, come è subito visto, man. tiene gli allineamenti. Tale corrispondenza è, perciò, una collineazione $\bar{\Phi}$, inerente un opportuno automorfismo di $F$, di $S_{3}^{\infty}$ in sè, nella quale alla congruenza $H(F, b, c)$ resta associata la congruenza $H\left(F, b^{\prime}, c^{\prime}\right)$. Tenuto presente che i concetti di quadrica e di congruenza lineare ellittica di rette sono invarianti per collineazioni e il fatto che una congruenza lineare ellittica di rette $\grave{e}$ individuata da quattro sue rette non appartenenti a una quadrica, possiamo affermare che la quadrica $x_{1} y_{2}-x_{2} y_{1}=0$ di $S_{3}^{\infty}$, poichè il campo $F$ ha ordine maggiore di tre, è mutata in sè dalla $\bar{\Phi}$ e, quindi, si verifica immediatamente che la $\bar{\Phi}$ trașforma la congrnenza lineare ellittica di rette $\vec{H}(F, b, c)$ nella congruenza lineare ellittica di rette $\bar{H}\left(F, b^{\prime}, c^{\prime}\right)$.

E appena necessario avvertire che il teorema precedente $\dot{\theta}$ applicabile per confrontare piani di HALL $S(F, b, c)$ e $S\left(F, b^{\prime}, c^{\prime}\right)$, relativi a un campo di caratteristica due poichè, essendo il campo $F$ perfetto, la congruenza $H(F, b, c)$ associata a $S(F, b, c)$ eे necessariamente ellittica.

Si noti, poi, che in [2] (Appendice II) è provato l'isomorfismo geometrico dei quasicorpi di HaLL relativi al campo di GaLors di ordine tre.

6. - Per completare i risultati annunciati nell'introduzione al lavoro, ci occuperemo, ancora, della determinazione del gruppo degli automorfismi spettante al piano di HaLL $S(F, b, c)$ relativo al campo $F$ e alla congruenza $H(F, b, c)$. Il teorema I (si tenga presente la dimostrazione della necessità delle condizioni poste) ei permette di affermare che ogni automorfismo di $S(F, b, c)$ (se $F$ ha almeno quattro elementi, se non $\dot{\theta}$ isomorfo a un suo sottocampo proprio, e se, avendo caratteristica due, è perfetto) '̀ realizzabile mediante un'opportuna affinità di $S_{4}$ (collineazione di $S_{4}$, inerente un automorfismo $\varphi$ di $F$, che muta $S_{3}^{\infty}$ in sè) la quale deve agire nello $S_{3}^{\infty}$ improprio di $S_{4}$ in modo da mutare in sè la congruenza $H(F, b, c)$; viceversa, ogni tale affinità di $S_{4}$ equivale a un automorfismo di $S(F, b, c)$. Ricordato che $H(F, b, c)$ si compone delle rette di $\bar{H}(F, b, c)$ non appartenenti alla quadrica $Q=x_{1} y_{2}-x_{2} y_{1}=0$ e delle rette di $Q$ che non siano elementi di $\bar{H}(F, b, c)$, possiamo enunciare il seguente:

Teorema II. - Sia $S(F, b, c)$ il piano di Hall relativo al campo $F$ e alla congruenza di rette $H(F, b, c)$ dello $S_{3}^{\infty}$ improprio dello $S_{4}$ lineare affine sopra il campo $F$; siano, inoltre, $\bar{H}(F, b, c)$ la congruenza lineare ellittica di rette di $S_{3}^{\infty}$ associala a $H(F, b, c)$ e $Q$ la quadrica $d i S_{3}^{\infty}$, di equazione $x_{1} y_{2}-x_{2} y_{1}=0$.

Se il campo $F$ ha almeno qualtro elementi, se non è isomorfo a un suo sottocampo proprio e, quindi, nel caso di caratteristica due, risulta $b \neq 0$, allora il gruppo degli automorfismi del piano di Hall $S(F, b, c)$ è isomorfo al gruppo delle affinità di $S_{4}$ che inducono nello $S_{3}^{\infty}$ una collineazione la quale muta in sè la congruenza lineare ellittica di rette $\bar{H}(F, b, c)$ e la quadrica $Q$. 


\section{BIBLIOGRAFIA}

[1] ANDRÈ, J., Über nicht-Desarguessche Ebenen mit transitiver Translationsgruppe, « Math. Z. *, $60(1954), 156.186$.

[2] Hall, M., Projective planes, "Trans. Amer. Math. Soc », 54, (1943) 229.277.

[3] LeNz, ${ }^{\circ} \mathrm{H}$., Kleiner Desarguesscher Sa'z und Dualitat in projektiven Ebenen, «Jahresbericht der Deutschen Math. ", (190̃4), 20-31.

[4] Lombardo-Radroe, L., Quelques résultats nouveaux et quelques problèmes ouverts dans la thèorie des quasicorps, Faculté des Sciences de Paris - Séminaire P. Dubreil, M.-L. Dubreil-Jacotin et $\mathrm{C}$. Pisot (Algebrè et Théorie des nombres) (1957.58).

[5] Panglta, G., Un insieme di piani ai traslazione isomorfi, Reticoli e Geometrie proiettive (Convegno Internazionale, Palermo-Messina, 1957) Cremonese, Roma, (1958), 109.119.

[6] Panelxa, G., Isomorfismo tra i piani di traslazione di Marshall Hall, « Rend. Acc. Naz. Lincei », 25 (1958), 172-173.

[7] Pickert, G., Projektive Ebenen, Springer, Berlin (1955).

[8] San-Soucre, Right altenative division rings of characteristic two, "Proc. Amer. Math. Soc. *, 6 (1955), 291.296.

[9] SegRE, B., Gli automorfismi del campo complesso ed un problema di Corrado Segre. - Rend. Acc. Naz. Lincei 3 (1947), 414.420.

[10] Skgre, B., Lezioni di Geometria moderna, vol. I, Zanichelli, Bologna, (1948). 